\title{
Structure, normal mode spectra, and mixing of a binary system of charged particles confined in a parabolic trap
}

\author{
W. P. Ferreira, ${ }^{1, *}$ F. F. Munarin, ${ }^{1}$ K. Nelissen, ${ }^{2}$ R. N. Costa Filho, ${ }^{1}$ F. M. Peeters,${ }^{2, \dagger}$ and G. A. Farias ${ }^{1}$ \\ ${ }^{1}$ Departamento de Física, Universidade Federal do Ceará, Caixa Postal 6030, Campus do Pici, 60455-760 Fortaleza, Ceará, Brazil \\ ${ }^{2}$ Department of Physics (U213), University of Antwerp (Campus Middelheim), Groenenborgerlaan 171, \\ B-2020 Antwerpen, Belgium
}

(Received 10 March 2005; revised manuscript received 5 May 2005; published 18 August 2005)

\begin{abstract}
We study the mixing of two different kinds of particles, having different charge and/or mass, interacting through a pure Coulomb potential, and confined in a parabolic trap. The structure of the cluster and its normal mode spectrum are analyzed as a function of the ratio of the charges (mass ratio) of the two types of particles. We show that particles are not always arranged in a shell structure. Mixing of the particles goes hand in hand with a large number of metastable states. The normal modes of the system are obtained, and we find that some of the special modes can be tuned by varying the ratio between the charges (masses) of the two species. The degree of mixing of the two type of particles is summarized in a phase diagram, and an order parameter that describes quantitatively the mixing between particles is defined.
\end{abstract}

DOI: 10.1103/PhysRevE.72.021406

PACS number(s): 82.70.-y, 36.40.Sx, 52.27.Lw

\section{INTRODUCTION}

In recent years there has been an increased interest in theoretical and experimental studies of two-dimensional (2D) confined clusters of classical particles. This is due to the wide applicability of such theoretical 2D models to real physical systems. For example, quantum dots are very well described by these simple models in the weak density limit and/or in the high magnetic field limit [1]. A classical approach to real systems is very convenient since many important systems can be modeled in this way. As examples of experimental realizations of such classical 2D systems, we can cite electrons on the surface of liquid helium [2,3], colloidal suspensions [4], ion traps [5], and strongly coupled dusty plasmas [6]. From a theoretical point of view, 2D charged confined clusters are a very interesting system which offers rich physics with several nontrivial effects (i.e., overcharging, phase transitions, Wigner crystallization, reentrant melting), and in which the reduced dimensionality and the finite size of the system allow often an "exact numerical" description.

In a parabolic confinement potential a $2 \mathrm{D}$ system of singly charged particles crystallizes in an ordered structure. For clusters with a small number of particles $(N)$, one observes the formation of a shell structure. When the number of particles increases, there is a competition between the internal triangular structure (i.e., Wigner crystal) and the circular confinement potential, which is responsible for the ring structure (i.e., leading to a 1D Wigner lattice at the edge) [7]. A Mendeleev-like table for these classical systems was presented in Ref. [7], and the normal mode properties of these systems were studied in Ref. [8]. A similar study for a Coulomb type confinement potential which corresponds to the classical analog of a 2D atom was presented in Ref. [9].

\footnotetext{
*Electronic address: wandemberg@fisica.ufc.br

†Electronic address: francois.peeters@ua.ac.be
}

Very recently, systems with two types of charged particles were studied $[10,11]$. The structure and melting of such classical clusters with the same mass were presented in Ref. [10]. It was shown that the particles arrange themselves in rings, with those of larger charge located at the border of the cluster. In addition, it was found that, for some special matching configurations, an intershell rotation was activated for temperatures higher than those observed in the cases of nonmatching configurations. The results presented in Ref. [10] were restricted to the special case in which the ratio between the two types of charges was $q_{1} / q_{2}=2$. In Ref. [11] a detailed study was made of the structure of clusters containing one or two impurities of different charge and mass as were realized in recent experiments [12]. References [10,11] did not address systems with a larger number of impurity particles, and did not study the normal modes of the system. As shown by Schweigert and Peeters [8], the frequencies of the normal mode spectrum can determine the melting temperature, and the particular intershell rotation mode (as the mode with minimum frequency) plays an important role in the melting properties of the system. When the intershell rotation mode is present the melting occurs in a two-step process with a very low temperature for angular disorder.

In Ref. [10] the authors mentioned that is possible to predict the appearance of the intershell rotation based only on the arrangement of the particles and on the ratio of the numbers of both types of particles in the cluster. However, we show here that there are other parameters that are more important than the configuration of the particles in determining the occurrence of the intershell rotation mode in the system. The ratio between charges and the ratio between masses of both types of particles are dominant parameters for the intershell rotation mode, and consequently for the melting.

Here we extend the results of Refs. $[10,11]$ and consider an arbitrary number of the two species of charged particles. The mixing of the two species of charges and their configurations are investigated as a function of the ratio between the charges and for different mass ratios. Concurrently, we also 
analyze the normal modes of the system, which give us information about the collective behavior of the particles and the stability of the configuration. Normal modes can be observed experimentally as shown recently in Refs. [13] and [14] for a 2D finite cluster and for a 1D chain of dust particles, respectively.

The paper is organized as follows. In Sec. II, we describe the model and the numerical procedure to find the minimum energy configurations and the frequencies of the normal mode spectra. The results for the structure as well as for the normal mode spectra of the system in the cases of different charges and masses of the two sets of particles are presented in Sec III. A phase diagram for mixing of the particles is constructed in Sec. IV. Our conclusions are given in Sec. V.

\section{THE MODEL}

Our system consists of a two-dimensional cluster with $N_{f}$ particles with fixed charge $q_{f}$ and fixed mass $m_{f}$, and $N_{v}$ particles with variable charge $q_{v}$ and/or variable mass $m_{v}$. All charged particles interact through a pure repulsive Coulomb potential $1 / r$, and the particles are kept together by a parabolic potential. The Hamiltonian of such a system is given by

$$
\begin{aligned}
H= & \sum_{i=1}^{N_{f}} \frac{1}{2} m_{f} \omega_{0}^{2} r_{i}^{2}+\sum_{i=1}^{N_{v}} \frac{1}{2} m_{v} \omega_{0}^{2} r_{i}^{2}+\frac{q_{f}^{2}}{\epsilon} \sum_{i>j=1}^{N_{f}} \frac{1}{\left|\mathbf{r}_{i}-\mathbf{r}_{j}\right|} \\
& +\frac{q_{v}^{2}}{\epsilon} \sum_{k>l=1}^{N_{v}} \frac{1}{\left|\mathbf{r}_{k}-\mathbf{r}_{l}\right|}+\frac{q_{f} q_{v}}{\epsilon} \sum_{m=1}^{N_{f}} \sum_{n=1}^{N_{v}} \frac{1}{\left|\mathbf{r}_{m}-\mathbf{r}_{n}\right|},
\end{aligned}
$$

where $\epsilon$ is the dielectric constant of the medium the particles are moving in, $m_{f}$ the mass of the particles with fixed charge, $m_{v}$ the mass of the particles with variable charge, and $r_{i}$ $\equiv\left|\mathbf{r}_{i}\right|$ the distance of the $i$ th particle from the center of the confinement potential. In order to reveal the important parameters of the system, it is convenient to define $q_{v} / q_{f}$ $=\alpha, m_{v} / m_{f}=\beta$ and to write the energy and the distances in units of $E_{0}=\left(m_{f} \omega_{0}^{2} q_{f}^{4} / 2 \epsilon^{2}\right)^{1 / 3}$ and $r_{0}=\left(2 q_{f}^{2} / m_{f} \epsilon \omega_{0}^{2}\right)^{1 / 3}$, respectively. In so doing, we obtain the dimensionless Hamiltonian

$$
\begin{aligned}
H= & \sum_{i=1}^{N_{f}} r_{i}^{2}+\beta \sum_{i=1}^{N_{v}} r_{i}^{2}+\sum_{i>j=1}^{N_{f}} \frac{1}{\left|\mathbf{r}_{i}-\mathbf{r}_{j}\right|}+\sum_{k>l=1}^{N_{v}} \frac{\alpha^{2}}{\left|\mathbf{r}_{k}-\mathbf{r}_{l}\right|} \\
& +\sum_{m=1}^{N_{f}} \sum_{n=1}^{N_{v}} \frac{\alpha}{\left|\mathbf{r}_{m}-\mathbf{r}_{n}\right|},
\end{aligned}
$$

which indicates that the system depends on the numbers of particles of both types $\left(N_{f}\right.$ and $\left.N_{v}\right)$, and on the parameters $\alpha$ and $\beta$. Note that $q_{f}$ and $m_{f}$ are the reference charge and mass, respectively. To obtain the ground state, we employed the Newton optimization technique after the standard Monte Carlo routine (Metropolis algorithm [15]). This procedure was outlined and compared with the standard Monte Carlo technique in Ref. [8]. In order to have sufficient confidence that we reached the ground state configuration, we considered typically 1000 different initial random configurations. However, for some values of $\alpha$ and $\beta(\alpha \approx \beta)$, where a large number of stable configurations $\left(N_{s c}\right)$ were observed $\left(N_{s c}\right.$ $\approx 160$ ), we considered more than 4000 initial random con- figurations. In this way, we found not only the ground state, but also the metastable states. From all the obtained stable states, the one with the lowest energy was taken as the ground state. To check if a configuration is stable, we calculated, using the Householder diagonalization technique, the eigenvalues of the dynamical matrix [8],

$$
H_{m n, i j}=\frac{\partial^{2} H}{\partial r_{m, i} \partial r_{n, j}},
$$

which give us the square of the frequencies of the normal modes of the system $(m, n=x, y$ and $i, j$ indicate the particle number). The configuration was taken as final when all frequencies squared of the normal modes were positive and real.

\section{RESULTS}

First we extend the results of Ref. [10] for $\alpha=2$ and $\beta$ $=1$ to an arbitrary $\alpha$. In addition, we also analyze the case in which the particles have different masses [i.e., $\beta \neq 1$ in Eq. (2)]. In general, the structure of the system is strongly dependent on the parameters $\alpha$ and $\beta$, and as will be show here, the two types of charged particles do not always separate into shells. In fact, the separation between the charges occurs for some critical values of $\alpha$ and $\beta$. Another important property of the system that depends on the parameters $\alpha$ and $\beta$ is the normal mode spectrum. Since the normal modes can reveal interesting features about the collective behavior of the particles and the stability of the configuration, thus will also be analyzed as a function of $\alpha$ and $\beta$.

\section{A. Variable charge}

\section{Structure}

In this section we take by default $\beta=1$. However, some results with $\beta \neq 1$ will also be commented on. The ground state configurations of clusters containing $N_{f}$ particles (black symbols) with fixed charge and $N_{v}$ particles (gray symbols) with variable charge are presented in Fig. 1 for different values of the parameter $\alpha$. As shown, a shell structure of the same type of particles is not always present, and its formation depends on $\alpha$, and on the numbers of particles of both types $\left(N_{f}, N_{v}\right)$. In general, there is a clear separation in shells between the two types of particles when $\alpha$ is distinct from 1 . In that case, the particles with largest charge are pushed toward the edge of the system and the inner part of the cluster contains the particles with the smallest charge. The ringlike configuration, as well as the general structure of the particles as a function of $\alpha$, is better observed by considering the distance of each particle $\left(r_{i}\right)$ to the center of the confinement potential as a function of $\alpha$. In general, there are two intervals of $\alpha$ in which particles of each type are separated into distinct shells forming perfect rings. This is shown in Fig. 2 for typical clusters where we took as examples the ones with $N_{f}=N_{v}=5$ particles and $N_{f}=7, N_{v}=6$ particles. Regions I, II, III, and IV separate the $\alpha$ intervals in which the cluster presents a shell structure from other $\alpha$ intervals characterized by a different ground state configuration. The vertical dotted 


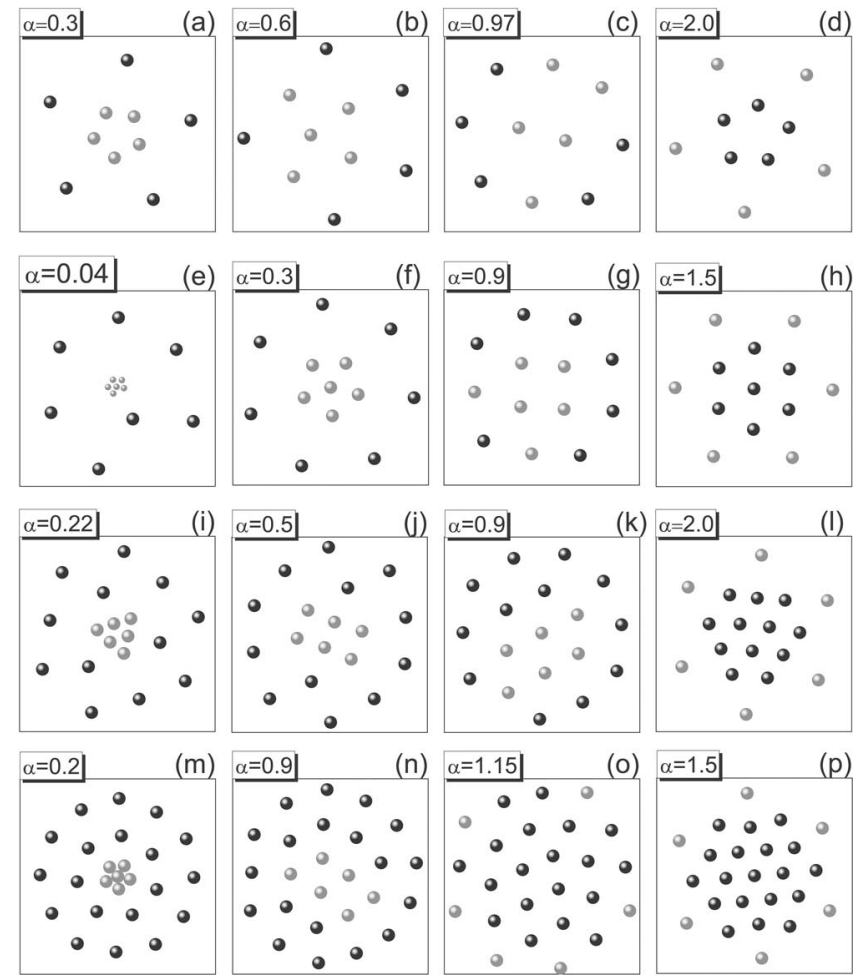

FIG. 1. Ground state configurations for clusters with different numbers of particles, and with different values for the parameter $\alpha$. The ratio between the masses is always $\beta=1$. The black spheres represent the $N_{f}$ particles with fixed charge, while the gray spheres represent the $N_{v}$ particles with variable charge. The numbers of particles in the figures are (a)-(d) $N_{f}=N_{v}=5$; (e)-(h) $N_{f}=7, N_{v}=6$; (i) - (l) $N_{f}=12, N_{v}=6$; and (m)-(p) $N_{f}=19, N_{v}=6$.

lines represent the change from the shell structure to another ground state configuration (and vice versa) through a first or a second order structural phase transition. The vertical dashed line in Fig. 2(b) indicates a transition from the shell structure to a different arrangement of the particles with no structural phase transition.

In Fig. 2(a) the perfect ringlike structure appears in regions I and III [see, e.g., Figs. 1(a) and 1(d)], while in Fig. 2(b) this is so in regions II and IV [Figs. 1(f) and 1(h)]. For $\alpha \approx 0.4$ [Fig. 2(b)] the rings start to be slightly deformed, i.e., broadened. In some cases, the ring structure disappears in the limit $\alpha \rightarrow 0$, as shown in Fig. 2(c), for the particular case of the cluster with $N_{f}=7$ and $N_{v}=6$ particles. Note that the particles with variable charge (gray symbols) behaves as one single effective particle in this limit.

The variation in the parameter $\alpha$ generates several modifications in the ground state configuration, which can be clearly noticed in Fig. 2, through the abrupt changes in the positions of the particles for specific values of $\alpha$. However, for some values of $\alpha$ the minimum energy configuration changes continuously but for which the derivative of the energy exhibits a discontinuity. Such changes are structural phase transitions, which can be classified as follows.

(1) When the ratio between charges and the ratio between masses are equal to unity (i. e., $\alpha=\beta=1$ ) there is an interchange of the positions of the particles with distinct charges.
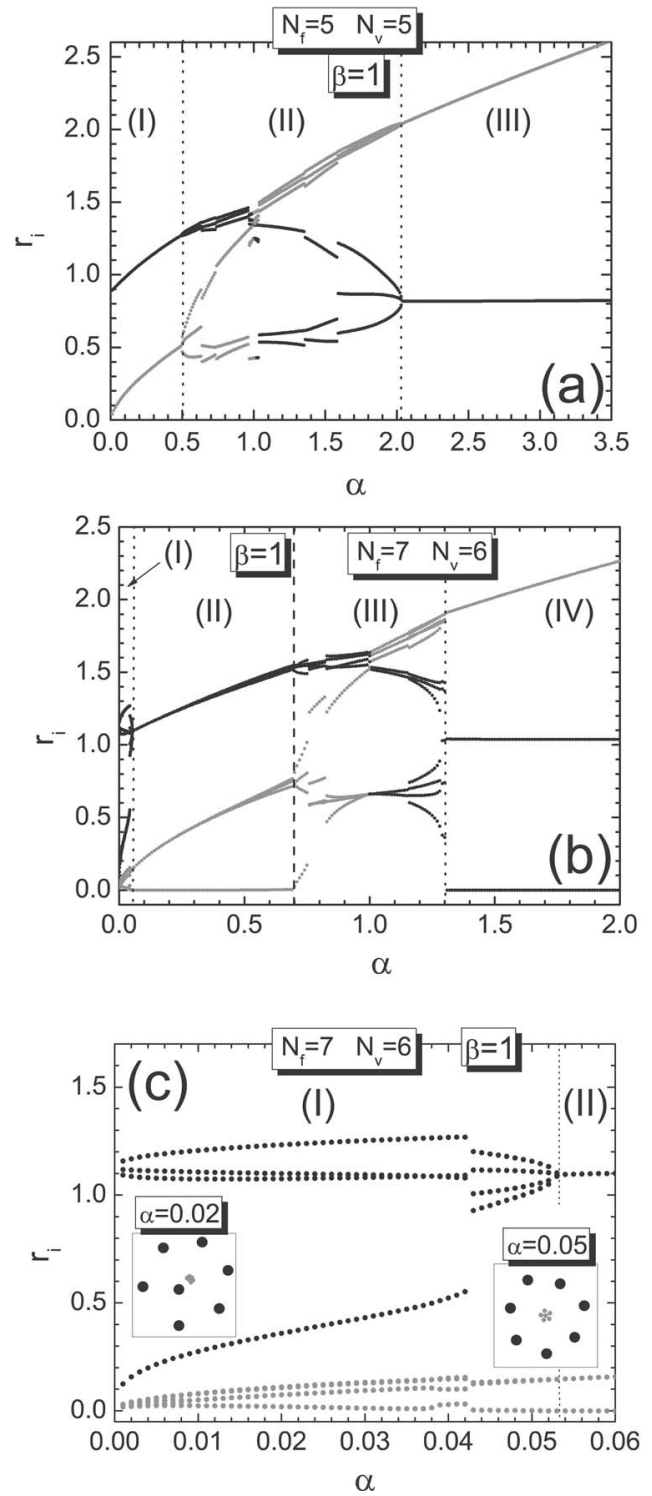

FIG. 2. Distance of each particle from the center of the confinement potential as a function of $\alpha$ for clusters with (a) $N_{f}=N_{v}=5$ particles, and with (b), (c) $N_{f}=7$ and $N_{v}=6$ particles. The black dots indicate the positions of one set of particles (i. e. $N_{f}$ ), while the gray curves represent the other set of particles (i.e., $N_{v}$ ).

Particles of one type that were located on the internal shell go to the external one, and vice versa (see Fig. 2). This changing is characterized by a first order structural phase transition, i.e., there is a discontinuity in the first derivative of the energy with respect to $\alpha$. If $\beta \neq 1$, no interchange in the position of the particles is observed for $\alpha=\beta$.

(2) First order structural phase transitions also occur for $\alpha \neq \beta$, when the positions of the particles abruptly change as a function of $\alpha$ (see Figs. 2 and 3).

(3) Transitions from region I to region II in Fig. 2(a), in which no jumps in the positions of the particles are present, correspond to a second order structural phase transition, which is characterized by a discontinuity in the second derivative of the energy with respect to $\alpha$ [Figs. 3(c)]. The first derivative is continuous [Fig. 3(b)]. 

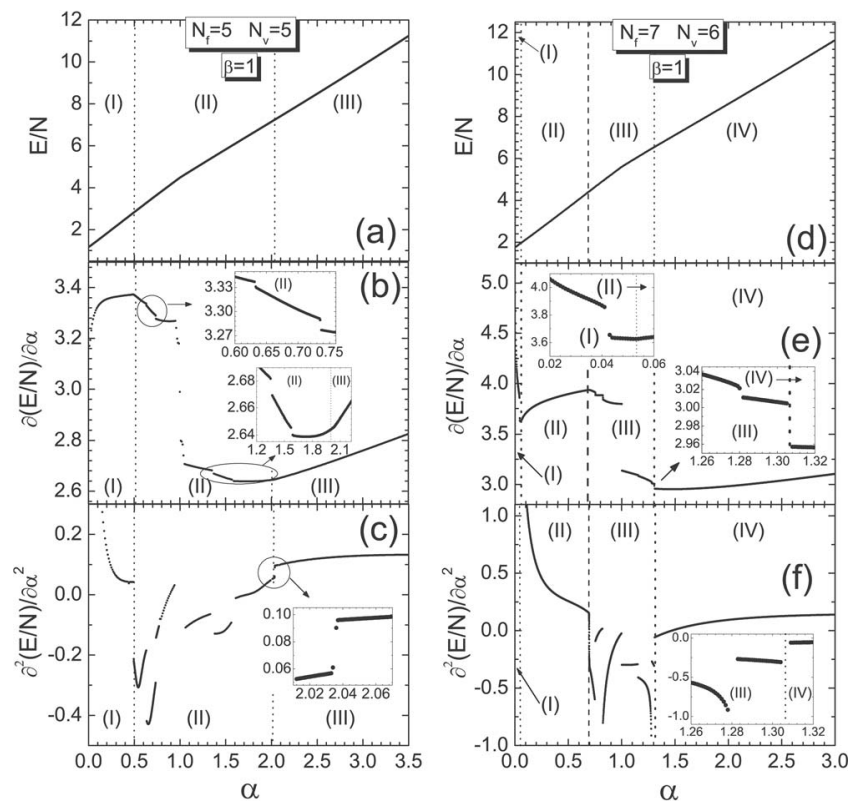

FIG. 3. (a), (d) Energy as a function of $\alpha$ for clusters with $N_{f}$ $=N_{v}=5$ and $N_{f}=7, N_{v}=6$ particles, respectively. The first and second derivatives of the energy as a function of $\alpha$ are also shown in (b), (e) and (c), (f), respectively. The vertical dotted (dashed) lines indicate the points where there are (are not) structural phase transitions between a ringlike configuration and a nonring configuration.

These features are also observed for the cases in which $\beta \neq 1$. In order to confirm such transitions, the energy curve as well as its first and second derivatives with respect to $\alpha$ are shown in Fig. 3, for the clusters with $N_{f}=N_{v}=5$ and $N_{f}$ $=7, N_{v}=6$ particles.

From Eq. (2), one expects that the energy of the system decreases with decreasing value of the parameter $\alpha$, as can also be observed in Figs. 3(a) and 3(d). In the cases where $N_{f}=N_{v}$, the $\alpha<1$ energy $\left(E_{\alpha<1}\right)$ is obtained from the $\alpha>1$ energy $\left(E_{\alpha>1}\right)$ through the scaling $E_{\alpha>1} \rightarrow E_{\alpha>1} \alpha^{4 / 3}$ and $\alpha$ $\rightarrow 1 / \alpha$. This scaling is also obvious from Eq. (2).

In the cases with $N_{f} \neq N_{v}$, a similar scaling for the $\alpha<1$ energy, from the $\alpha>1$ energy, is possible through the relations $E \rightarrow E \alpha^{n}$ and $\alpha \rightarrow 1 / \alpha$, but now the exponent $n$ depends on the number of both types of particles $\left(N_{f}, N_{v}\right)$. For example, for the cluster with $N_{f}=7, N_{v}=6$ particles we found $n=1.23$. For $N_{f} \neq N_{v}$ there is no simple way to obtain this scaling directly from Eq. (2).

For $\alpha \approx 1$ a clear mixing occurs, i.e., particles of both types are not separated in distinct shells [e.g., Figs. 1(c), $1(\mathrm{~g})$, and $1(\mathrm{k})$, . In that case, a large number of stable configurations with different energy are present, as can be observed in Figs. 4(b) and 4(e). For $\alpha=\beta=1$ the number of stable states suddenly decreases because the particles are now identical and mixing becomes irrelevant. The number of stable states presents the same qualitative dependence on the parameter $\alpha$ in all studied cases. A very interesting feature of the present system can also be observed in Fig. 4: a maximum value of the number of stable states is reached when the ratio between the charges is approximately equal to the ratio between the masses $(\alpha \approx \beta)$. This maximum is larger for $N_{f} \neq N_{v}$, and its appearance is a consequence of the near degeneracy of the configurations which differ only by an interchange of, e.g., two particles of different type.

Concerning the structure of the system, it is also interesting to notice the lack of rotational symmetry of the minimum energy configuration observed in some cases [i.e., Figs. 1(b), $1(\mathrm{e}), 1(\mathrm{~g})$, and $1(\mathrm{k}))]$. Ground state configurations, which present rotational symmetry, are observed in parabolic confined clusters with a small number of single charged particles [7], and this is a consequence of the symmetry of the confinement potential. However, in the present case, the positional correlations between the particles with distinct charges overcome the circular symmetry of the parabolic confinement potential, resulting in asymmetrical configurations. The presence of particles with different charges strongly affects the structural properties of the system.

\section{Normal modes}

As an example, we present in Fig. 5 the frequencies of the normal modes as a function of $\alpha$ for a cluster with $N_{f}=7$ and $N_{v}=6$ particles. The frequencies corresponding to the center of mass mode $(\mathrm{CM})\left(\omega / \omega_{0}=\sqrt{2}\right)$, the breathing mode $(\mathrm{BM})$ $\left(\omega / \omega_{0}=\sqrt{6}\right)$, and the mode with frequency $\omega / \omega_{0}=0 \mathrm{do}$ not depend on the parameter $\alpha$, and such a behavior is observed in all studied cases with $\beta=1$. This fact is a special feature of the parabolic confinement potential when there exist power law forces between the particles, which can be verified analytically $[16,17]$. However, for $\beta \neq 1$, which means particles with distinct masses, there is a symmetry breaking in the confinement potential [see Eq. (2)], which results in the disappearance of the $\mathrm{CM}$ and $\mathrm{BM}$.

In general, the normal mode frequencies exhibit jumps at first order structural phase transitions (with exception of the case $\alpha=\beta=1$ ). In the case of second order structural phase transitions no jumps in the normal mode frequencies are observed, but the lowest nonzero frequency goes to zero at the transition point. that is there is a softening of the minimum frequency mode in this case.

The vertical dashed lines in Fig. 5 divide the $\alpha$ interval into four regions (I, II, III, IV) associated with the arrangement of the particles in the cluster, as previously defined in Fig. 2(b). In the particular regions II $(0.053 \leqslant \alpha<0.694)$ and IV $(\alpha \geqslant 1.309)$ the particles with different charges are separated into distinct rings. In such regions, the normal mode frequencies present a larger degeneracy than that observed in regions I $(\alpha \leqslant 0.053)$ and III $(0.694 \leqslant \alpha<1.309)$, and this is associated with the higher symmetry of the ground state configuration. In region IV some modes have frequencies that become independent of $\alpha$, while for other ones the normal mode frequencies vary slowly as a function of $\alpha$.

Now we carefully analyze the behavior of the lowest nonzero frequency (LNF) and its associated normal mode, which are associated with the stability of the ground state configuration. In addition, the normal mode associated with the LNF plays an important hole in determining the melting properties of the cluster [8]. In Fig. 6 the LNF of the cluster with $N_{f}$ $=7, N_{v}=6$ particles is presented as a function of $\alpha$. This particular cluster is considered because it can present commensurate (when the number of particles on the outer shell can be written as an integer multiple of the number of par- 

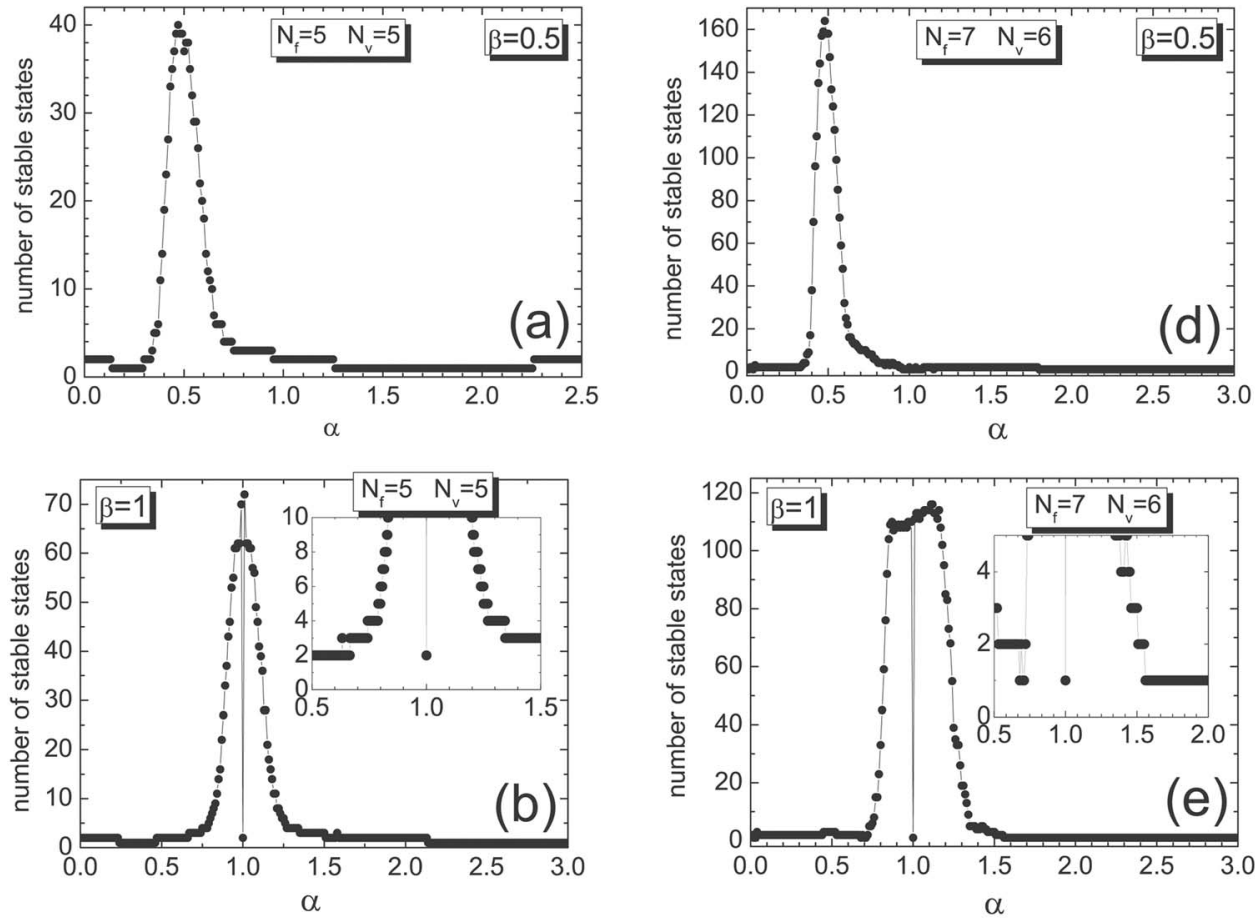

FIG. 4. Number of stable configurations as a function of $\alpha$ for clusters with $N_{f}=N_{v}=5$ and $N_{f}$ $=7, N_{v}=6$ particles and different ratio between the masses of the two different types of particles $(\beta=0.5,1,2)$.
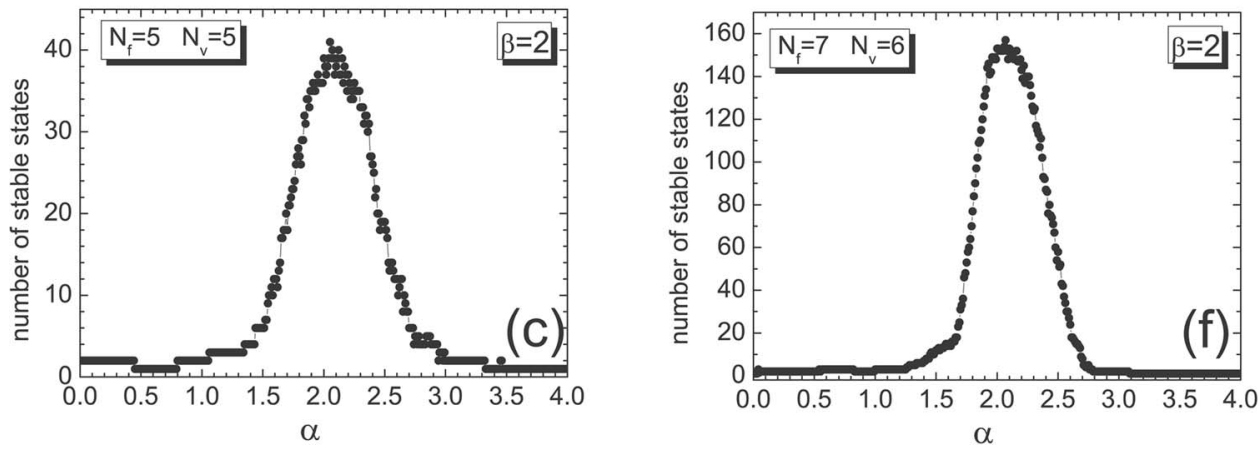
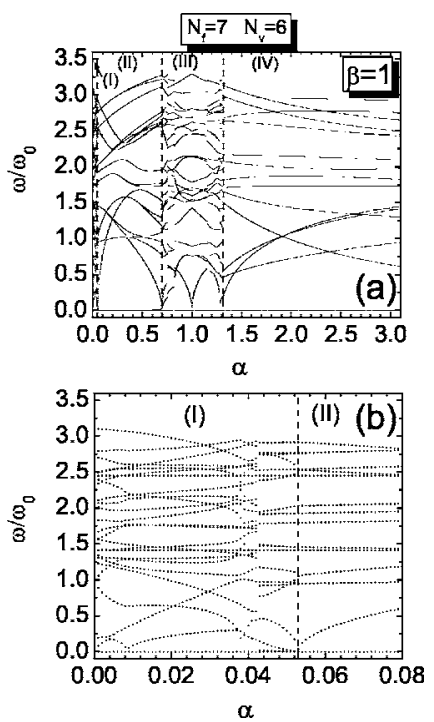

FIG. 5. (a) Normal mode frequencies as a function of $\alpha$ for a cluster with $N_{f}=7$ and $N_{v}=6$ particles. (b) Normal mode frequencies at region $\mathrm{I}$ for the same cluster. ticles on the inner shell) or incommensurate configurations depending on the values of $\alpha$ and $\beta$. We will analyze the behavior of the LNF in such cases. The same regions defined in Fig. 5 are also indicated. However, new subregions (distinguished by dotted lines) separating different $\alpha$ intervals in which the ground state configuration is the same, but the excited normal mode is different (regions IVa, IVb, IVc), are also defined. In region II $(0.053 \leqslant \alpha<0.694)$, the ground state configuration and the lowest normal mode are shown as insets. Particles with smaller charge (gray spheres) are located in the internal region of the cluster, while the other particles (black spheres) are placed on the external ring. The system presents an incommensurate configuration $(1,5,7)$ (which means one particle on the center of the trap, five particles on the first ring, and seven particles on the external ring), since the number of particles on the outer shell is not an integer multiple of the number of particles on the inner shell. In regions (IV) the particles are also arranged in three shells, but now in a commensurate configuration $(1,6,6)$ in which all particles with greater charge are pushed to the external shell.

In the present system we observe that the lowest normal mode is strongly dependent on the parameter $\alpha$. In regions IVa, IVb, and IVc of Fig. 6(a), for example, in spite of the 

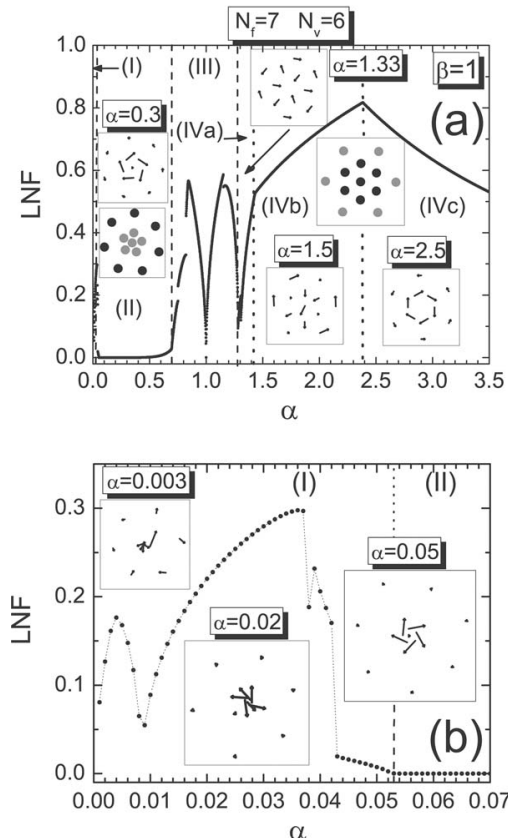

FIG. 6. (a) The lowest nonzero frequency (LNF) as a function of $\alpha$ for a cluster with $N_{f}=7$ and $N_{v}=6$ particles. (b) The LNF at region I for the same cluster.

same configuration of the cluster, the associated lowest normal mode is tuned by the value of $\alpha$. In the $\alpha$ interval $1.43 \lesssim \alpha \lesssim 2.38$ (region IVb the lowest normal mode corresponds to a motion as in a vortex-antivortex pair, while for $\alpha>2.38$ (region IVc it is an intershell rotation(ISR) [see insets of Fig. 6(a)]. This fact shows that the potential barrier for the ISR mode is not uniquely controlled by the ratio of the number of particles on the shells, as mentioned in Ref. [10], but first by the parameter $\alpha$, which controls the potential barrier formed by the two types of particles. However, when the intershell rotation is the lowest normal mode, the frequency for activation of such a mode is strongly dependent on the ratio of particles on the shells. For example, in region II, since the particles are arranged in an incommensurate configuration, the LNF corresponding to the ISR mode is very small $\left(\omega_{\min } \sim 10^{-4}\right)$. On the other hand, in regions IV the particles are arranged in a commensurate configuration, and the excitation frequency for the normal mode (ISR) is very large $\left(\omega_{\min } \sim 0.5-0.8\right)$ compared with the frequency for the ISR mode in region II. A similar dependence of the LNF with respect to the number of particles on the distinct shells, in a system with one type of charged particle, was observed by Schweigert and Peeters [8].

As mentioned before, in region IVc the ISR is the corresponding lowest normal mode. As can be seen, the associated LNF decreases with increasing value of $\alpha$. Such a behavior is understood through the effective coupling between the adjacent shells. The repulsive interaction between particles with variable charge increases with increasing value of $\alpha$. As a consequence, the radius of the external ring becomes larger. Since the radius of the internal ring does not change in region IV [see Fig. 2(b)], the distance between the adjacent shells becomes larger too. This fact makes the interaction between particles located in adjacent shells weaker, and the energy necessary to excite the ISR smaller. Such a behavior is observed in all cases in which the particles are separated in different rings, and when the ISR mode is present. The dependence of the value of the frequency associated with the intershell rotation mode on the parameter $\alpha$ is another particular feature of the present system. As commented before, since the frequency of the intershell rotation mode (as the mode with minimum frequency) plays an important role in the melting properties of the system [8], we can conclude that the ratio between the charges of both types of particles should be also an important parameter to determine the melting properties of the cluster, and this is an interesting direction for further analysis of the present model system.

In the intermediate region III $(0.694 \leqslant \alpha<1.308)$, the ground state configurations are mixed, i.e., the particles with different charges are not completely separated in distinct shells [e.g., Fig. $1(\mathrm{~g})$ ]. With the exception of the case $\alpha=1$, the normal modes do not correspond to the ISR. Instead, the particles oscillate collectively around their equilibrium position like in a vortice-antivortice pair.

\section{B. Two species with different mass}

\section{Structure}

Now we will present the results for the case in which the system described by Eq. (2) consist of particles with different masses. The first type has mass $m_{f}$, while the second type has mass $m_{v}$ which we shall vary. The ratio between the masses is given by the parameter $\beta=m_{v} / m_{f}$. Here, we consider two cases for the ratio between the charges of the two types of particles, namely, $\alpha=1$ and 2.

In Fig. 7 examples for the structure of clusters with different values of the parameter $\beta$ are shown. For small and large values of $\beta$ the cluster configuration is characterized by a separation of the particles into different shells. Particles with smaller mass are pushed out toward the external shells. Such a feature is due to the fact that particles with larger mass move to the internal region of the cluster in order to minimize the confinement energy [see Eq. (2)]. An interplay between the electrostatic repulsion between the negative particles, and the confinement strength determines the minimum energy configuration. For some values of $\beta$, and in spite of the circular symmetry of the confinement potential, we notice that the system can be stabilized in a configuration without any rotational symmetry, as shown, e.g., in Figs. 7(b), $7(\mathrm{c}), 7(\mathrm{j})$, and $7(\mathrm{k})$.

In order to better observe how the the ground state configurations is influenced by the parameter $\beta$, we investigate the distance of each particle from the center of the confinement potential $\left(r_{i}\right)$ as a function of $\beta$. This is shown in Fig. 8, where we took as example typical clusters with $N_{f}=N_{v}=5$ and $N_{f}=6, N_{v}=5$ particles. Different regions (I, II, and III), which are associated with distinct ground state configurations, are indicated in the figure. For the cluster with $N_{f}$ $=N_{v}=5$ particles [Fig. 8(a)], a perfect ringlike configuration, similar to the ones in Figs. 7(a) and 7(d), is observed in regions I and III, respectively. In the intermediary region II, different configurations are present [e.g., see Figs. 7(b) and $7(\mathrm{c})]$. The transitions from region I to region II and from 


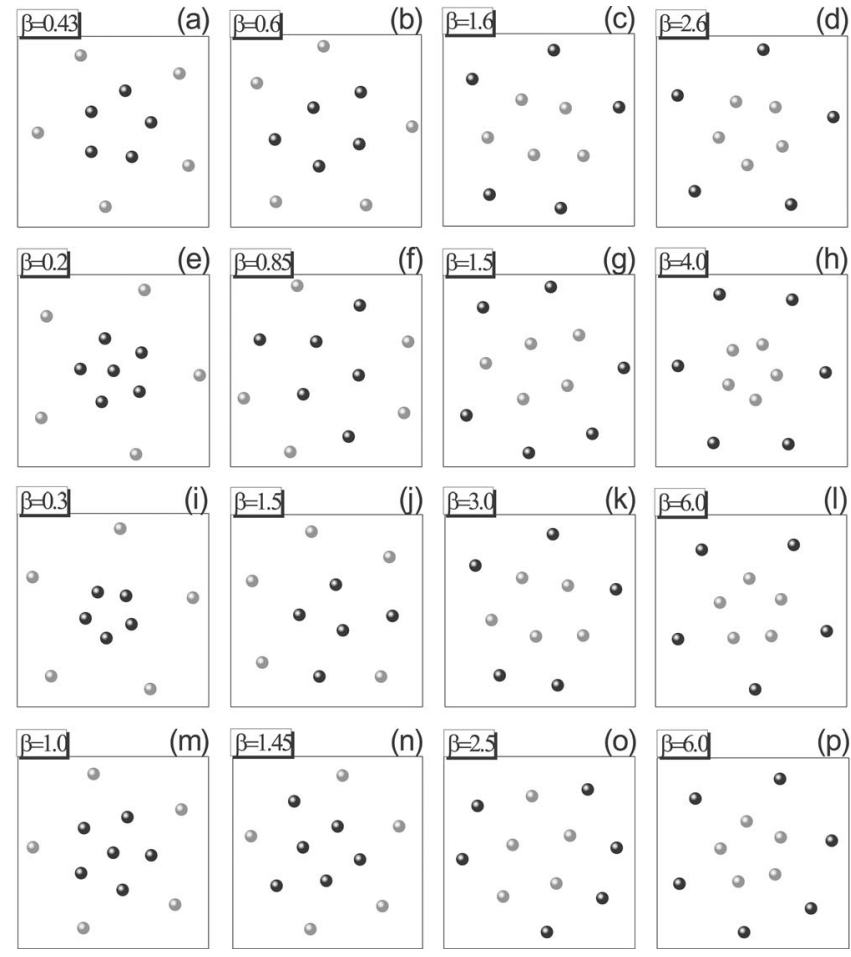

FIG. 7. Ground state configurations for clusters with different number of particles and different mass ratios. The black spheres represent the $N_{f}$ particles with fixed mass, while the gray spheres represent the $N_{v}$ particles with variable mass. In (a)-(d) and (i)-(l) we consider the cluster with $N_{f}=N_{v}=5$ particles, while (e)-(h) and (m)-(p) are clusters with $N_{f}=6, N_{v}=5$ particles. The value for the parameter $\beta$ is indicated in the figures. In (a)-(h) the ratio between the charges is $\alpha=1$, while in (i)-(p) we consider $\alpha=2$.

region II to region III occur in such a way that the positions of the particles change continuously. This is a typical characteristic of a second order structural phase transition. This fact is confirmed in Figs. 9(b) and 9(c), in which the first and second derivatives of the energy with respect to $\beta$ are shown. Note that at the transition points between regions I and II ( $\beta=0.419)$, and between regions II and III $(\beta=2.386)$, a discontinuity is observed only in the curve for the second derivative. The first derivative presents no discontinuity.

For some values of $\beta$ there is an abrupt modification of the ground state configuration, which is associated with a first order structural phase transition. When $\alpha=1$, the first order structural phase transition associated with the point $\beta$ $=\alpha$ is characterized by an inversion of the positions of both types of particles. For $\alpha \neq 1$, the point $\beta=\alpha$ does not correspond to an inversion of the positions of the particles with different masses. In general, for $\beta<1$ particles with fixed mass are located at the internal region of the cluster, while for $\beta>1$ such particles are pushed toward the edge of the system [Fig. 8(a)]. Such a behavior was observed in clusters with distinct values for $N_{f}$ and $N_{v}$ [e.g., Fig. 8(b)].

The total energy as a function of $\beta$ is shown in Fig. 9(a). As in the case of particles with different charges, the $\beta<1$ energy $\left(E_{\beta<1}\right)$ can be obtained from the $\beta>1$ energy $\left(E_{\beta>1}\right)$, through the scaling $E_{\beta>1} \rightarrow E_{\beta>1} \beta^{-1 / 3}$ and $\beta \rightarrow 1 / \beta$, which is easily obtained from Eq. (2) by inspection. This is valid only
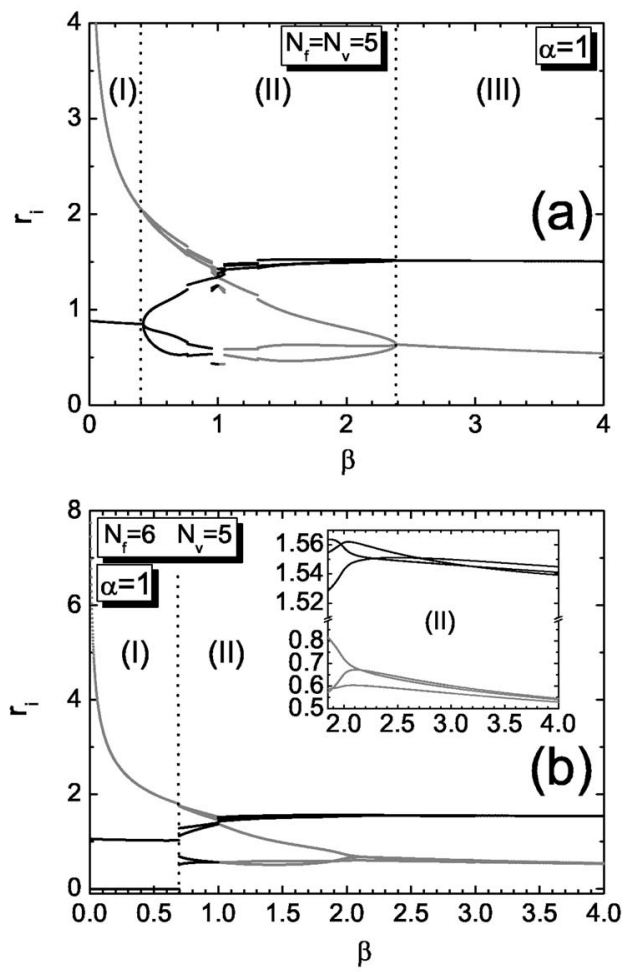

FIG. 8. Distance of each particle from the center as a function of $\beta$ for clusters with (a) $N_{f}=N_{v}=5$ particles, and (b) $N_{f}=6$ and $N_{v}$ $=5$ particles. The black curves indicate the positions of one set of particles (i.e., $N_{f}$ ), while the gray curves represent the other set of particles (i.e., $N_{v}$ ).

if $N_{f}=N_{v}$. When $N_{f} \neq N_{v}$, we can find similar relations numerically, namely, $E_{\beta>1} \rightarrow E_{\beta>1} \beta^{-n}$ and $\beta \rightarrow 1 / \beta$, but now the exponent $n$ depends on $N_{f}$ and $N_{v}$. For example, for the cluster with $N_{f}=6, N_{v}=5$ particles we found $n \approx 0.297$, while for the cluster with $N_{f}=7, N_{v}=6$ particles we found $n \approx 0.303$.

For the cluster with $N_{f}=6, N_{v}=5$ particles [Fig. 8(b)] a perfect ringlike structure [Fig. $7(\mathrm{e})]$ is observed only in region I. In the interval $\beta>1$ no ring structure, in which all particles in a ring are placed at the same distance from the center of the confinement potential, is observed [see the inset in Fig. 8(b)]. However, the particles with different masses are still separated in distinct shells. Because of the noncommensurate number of particles on both shells, a ground state configuration with perfect rings cannot be obtained, at least for the considered values of $\beta$. As shown in Fig. 8(b), the positions of the particles change fast but continuously with $\beta$ at $\beta \approx 2.0$. From Figs. 9(e) and 9(f), it is clear that no structural phase transition is observed in the system. The $\lambda$-like shape of the derivative $\partial^{3} E / \partial \beta^{3}$ [see the inset in Fig. 9(e) and 9(f)], is similar to the so called continuous transition, previously found in Ref. [18] for the case of two coupled classical dots.

The transition from region I to region II is characterized by an abrupt change of the positions of the particles. Such a modification is associated with a first order structural phase transition [Fig. 9(e)]. This behavior is different from the one found in the case $N_{f}=N_{v}=5$, where a second order structural transition is observed when the system changes from the 

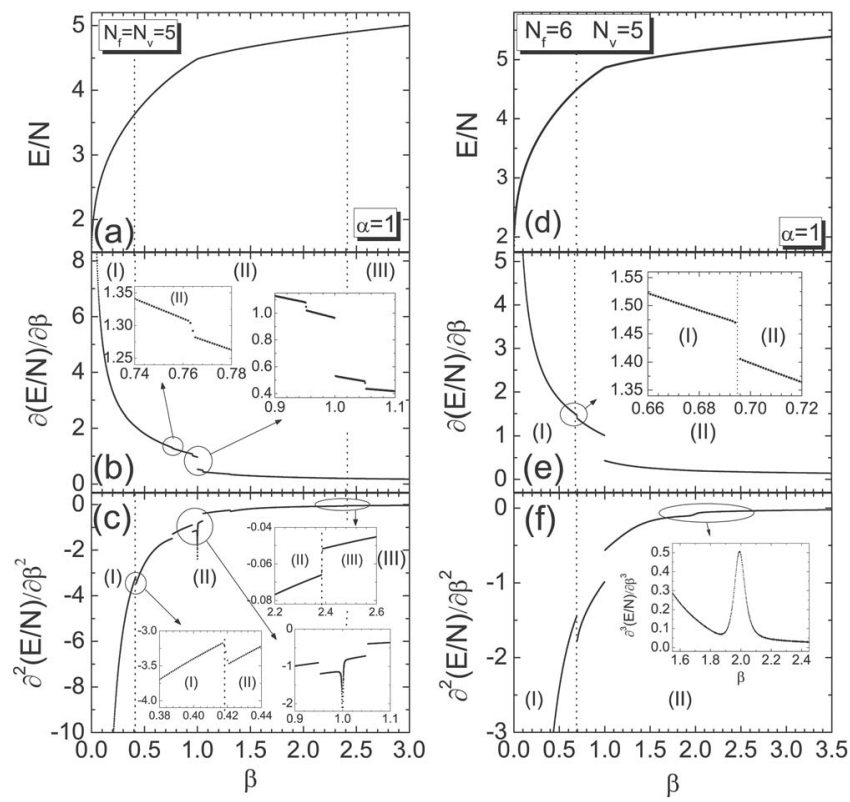

FIG. 9. (a) Total energy per particle as a function of $\beta$ for a cluster with $N_{f}=N_{v}=5$ particles. (b), (c) The first and second derivatives of the energy with respect to $\beta$. (d) Total energy per particle as a function of $\beta$ for a cluster with $N_{f}=6, N_{v}=5$ particles. (e), (f) The first and second derivatives of the energy with respect to $\beta$ for the same cluster. The third derivative of the energy with respect to $\beta$ is shown as an inset in (f).

ringlike configuration to the nonring one [Fig. 9(c)]. Second order structural phase transitions were not observed in the cluster with $N_{f}=6, N_{v}=5$ particles. In general, we found that the order of the structural phase transition depends on the parameters $\alpha$ and $\beta$, and on the number of particles of both types, namely, $N_{f}$ and $N_{v}$.

As shown in the previous case of particles with different charges, a similar maximum in the number of stable configurations is also observed as a function of $\beta$ when the particles have distinct masses. More specifically, a maximum in the number of stable states is present when the ratio between the masses is not exactly equal, but approximately equal to the ratio between the charges $(\beta \approx \alpha)$. In the particular case $\alpha$ $=1$, all particles have the same charge and the same mass, and the number of stable states is equal to the number of stable states of the system with $\left(N_{f}+N_{v}\right)$ identical particles. In Fig. 10 the number of stable configurations is given as a function of $\beta$ where we took again as example clusters with $N_{f}=N_{v}=5$ and $N_{f}=6, N_{v}=5$ particles. Note that in the cases in which the particles also have different charges $(\alpha \neq 1)$, the maximum in the number of stable configurations is slightly shifted from the value $\beta=\alpha$ [see Figs. 10(a), 10(c), 10(d), and 10(f)]. The maximum in the number of stable states is slightly shifted to the left (with respect to the point $\alpha=\beta$ ) when $\alpha$ is increased, and slightly shifted to the right when $\alpha$ is decreased.

\section{Normal modes}

In Fig. 11 the normal mode frequencies, as a function of $\beta$, for the clusters with $N_{f}=N_{v}=5$ and $N_{f}=6, N_{v}=5$ particles
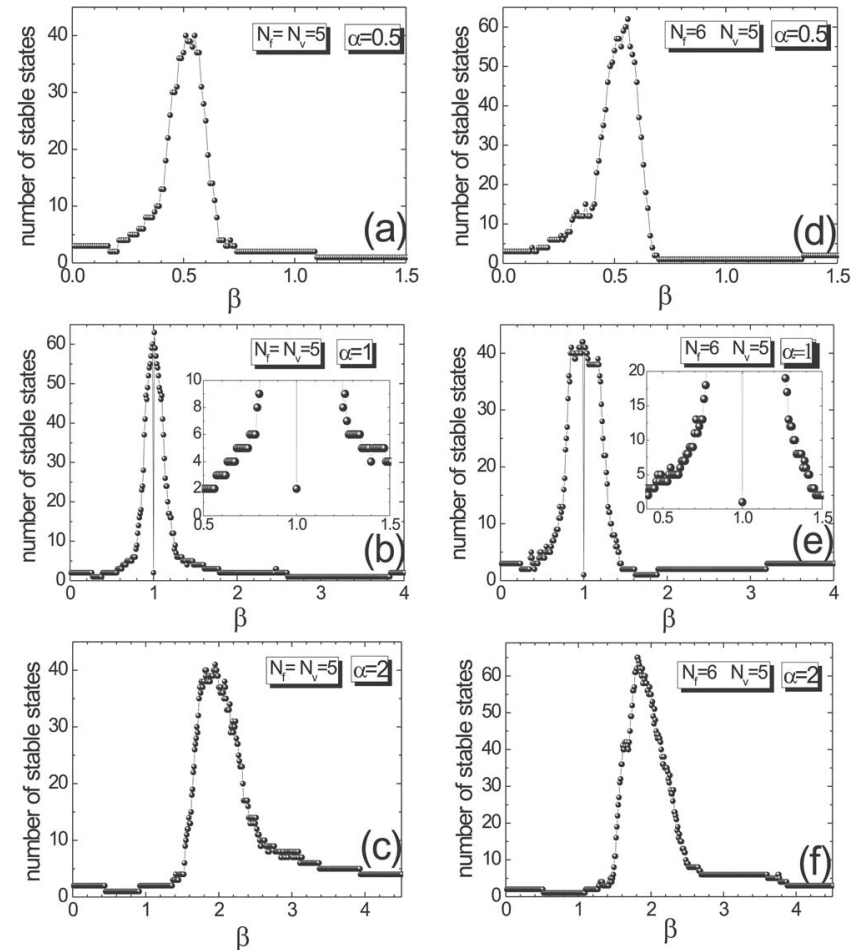

FIG. 10. Number of stable configurations as a function of $\beta$ for clusters with $N_{f}=N_{v}=5$ and $N_{f}=6 ; N_{v}=5$ particles and different ratios between the charges of the two different types of particles $(\alpha=0.5,1,2)$.
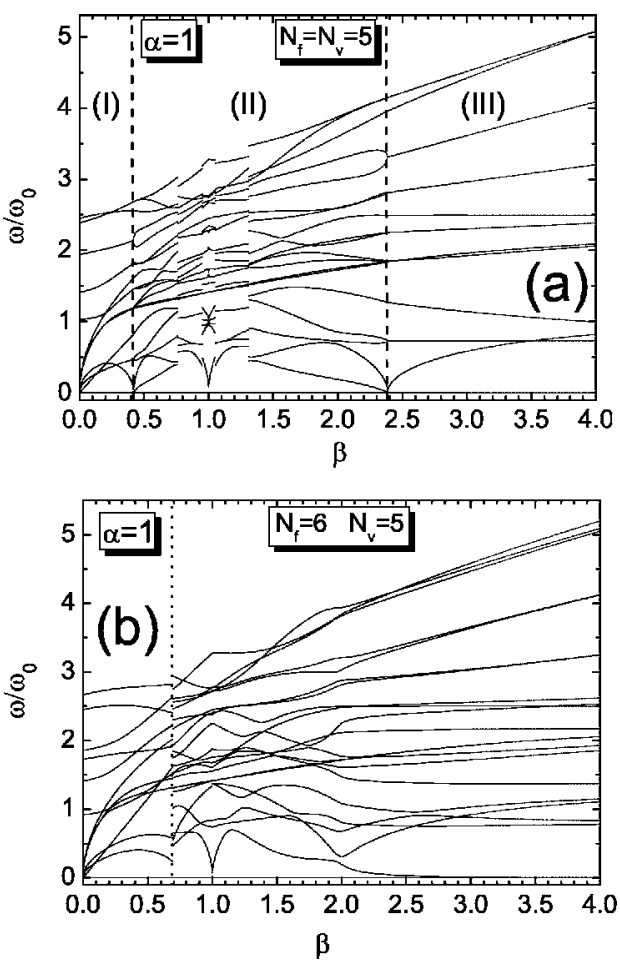

FIG. 11. Normal mode frequencies as a function of $\beta$ corresponding to the ground state configuration of clusters with (a) $N_{f}$ $=N_{v}=5$ particles and (b) $N_{f}=6$ and $N_{v}=5$ particles. 


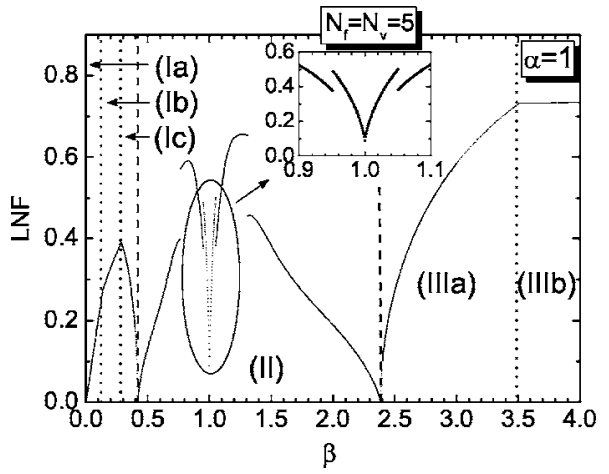

FIG. 12. Lowest nonzero frequency as a function of $\beta$ for clusters with $N_{f}=N_{v}=5$ particles. Regions I and III separate the $\beta$ intervals in which the system exhibits the ring configuration. In region II different configurations are observed. The subregions Ia, Ib, Ic, IIIa, and IIIb separate the $\beta$ intervals in which the same ground state configuration is observed, but where distinct normal modes are present.

are shown. These clusters are interesting because they present commensurate or incommensurate configurations for different values of $\alpha$ and $\beta$. The same regions defined in Fig. 8 are again considered. In general, the frequencies increase with increasing value of $\beta$. With the exception of the mode corresponding to the rotation of the entire cluster around the $z$ axis $(\omega=0)$, the frequencies of all other modes depend on $\beta$. However, for large values of $\beta$ some modes have frequencies which become independent on $\beta$. The center of mass and the breathing modes, which are present in a parabolic confined system with one type of particle, are not observed in the present system, because the confinement potential is no longer the same for all particles. Such a symmetry breaking in the confinement potential destroys the center of mass mode and the breathing mode.

In the $\beta$ intervals corresponding to the more symmetrical ring configuration (regions I and III for the cluster with $N_{f}$ $=N_{v}=5$ particles, and region I for the cluster with $N_{f}$ $=6, N_{v}=5$ particles), most of the normal mode frequencies are degenerate.

The normal mode frequencies are affected by structural phase transitions, and they can be used to identify such transitions. Similar general features for first and second order structural phase transitions observed in the case with variable charge are also present in the case of particles with distinct masses.

As a general rule, we observed that the normal modes are mainly determined by the ratio between masses, and to a lesser extent by the number of particles $N_{f}$ and $N_{v}$. This behavior could be confirmed in clusters with different number of particles, and with different ratios between the charges of the two types of particles $(\alpha)$. Such a behavior can be clearly confirmed by the analysis of the LNF.

The LNF gives us information on the stability and the deformability of the cluster configuration. As an example of the obtained results, the LNF as a function of $\beta$, for the $N_{f}$ $=N_{v}=5$ cluster, is shown in Fig. 12. The same regions defined in Fig. 8(a) are considered again (regions separated by vertical dashed lines). However, in order to better understand
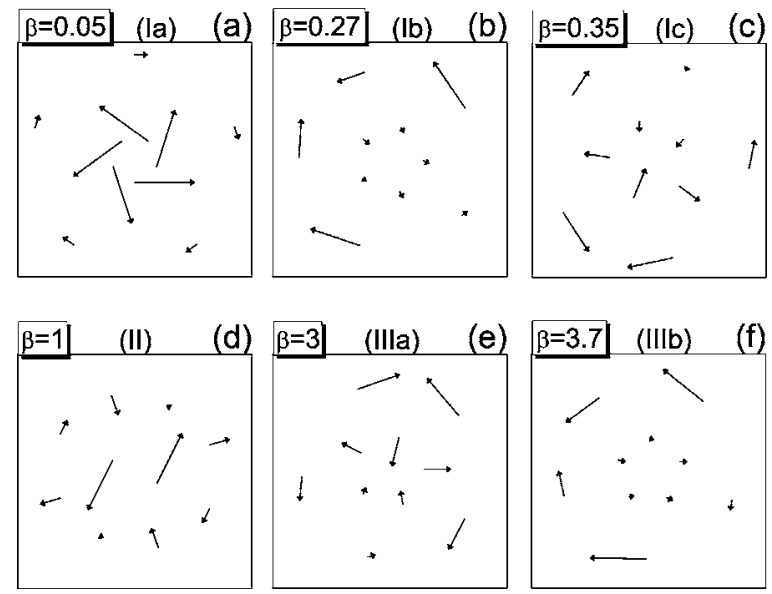

FIG. 13. Normal modes for the cluster with $N_{f}=N_{v}=5$ particles and $\alpha=1$. In each figure the subregions defined in Fig. 12 are indicated.

the results, additional subregions are also defined (distinguished by vertical dotted lines). Such subregions separate the cases in which the cluster presents the same ground state configuration, but distinct normal modes.

In region Ia $(\beta \leqslant 0.136)$, Fig. 12 , the particles are excited in the intershell rotation mode [Fig. 13(a)]. By changing the parameter $\beta$, the ground state configuration remains the same, but the excited mode is changed to the one observed in region $\mathrm{Ib}(0.136<\beta \leqslant 0.284)$ [Fig. 13(b)]. A further increase in the parameter $\beta$ leads the system to region Ic $(0.284<\beta$ $\leqslant 0.419$ ), with a different normal mode [Fig. 13(c)], but the particles are still arranged in the same ground state configuration [Fig. 7(a)]. These results show that the potential determining the frequency of the LNF is mainly determined by the parameter $\beta$.

In region II $(0.419<\beta \leqslant 2.385)$ different minimum energy configurations [Fig. 8(a)], as well as different normal modes are found. In Fig. 13(d), the particular case $\beta=1$ presents the same normal mode observed in a single cluster with $N=10$ particles, as it should.

For $\beta=2.385$ there is a second order structural phase transition to region IIIa $(2.385<\beta \leqslant 3.5)$, in which the system is again a perfect ringlike configuration [Fig. 7(d)]. The associated normal mode is presented in Fig. 13(e). For $3.5<\beta$ $\leqslant 7.32$ the system reaches region IIIb with the same ground state configuration, as observed in region IIIa, but with a different normal mode, which corresponds to the one shown in Fig. 13(f). Finally, when $\beta>7.32$ (not shown in Fig. 12) the system still remains in a perfect ring-like configuration, but the lowest normal mode changes to the ISR mode.

As in the case for variable charge, and for all analyzed cases with variable mass, we observed that when the particles are arranged in a commensurate configuration, the associated ISR is three to four orders of magnitude larger than the frequency of the same mode of a noncommensurate configuration. However, we note here that the effective potential determining the frequency of the ISR mode can be tuned by the parameters $\alpha$ and $\beta$, and consequently, the stability of the ground state configuration is tuned in this way. 

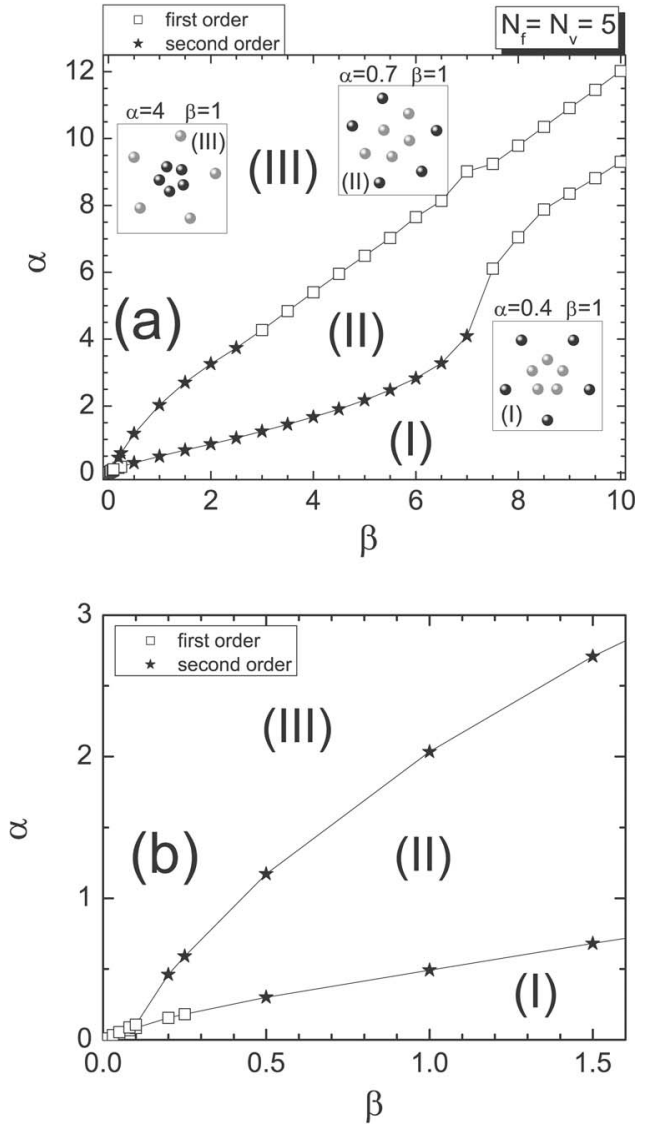

FIG. 14. Phase diagram: the ratio between the charges $\alpha$ as a function of the ratio between the masses $\beta$ for the cluster with $N_{f}$ $=N_{v}=5$ particles. An enlargement is shown in (b). The insets in (a) show the ground state configurations for some points $(\alpha, \beta)$.

\section{THE PHASE DIAGRAM FOR MIXING}

From the previously obtained results, we learned that the structure of the cluster is strongly dependent on the ratio between charges $(\alpha)$ and/or the ratio between masses $(\beta)$ of both type of particles. For specific values of $\alpha$ and/or $\beta$, the different type of particles separate in distinct shells.

The degree of mixing of the two species of particles can be summarized in a $(\alpha, \beta)$ phase diagram. This is presented in Figs. 14 and in 15 for the previously discussed clusters with $N_{f}=N_{v}=5$ and $N_{f}=7, N_{v}=6$ particles, respectively. The phase diagrams are divided in different regions (I, II, III, and IV) by lines (and symbols), which are defined here as the transition lines where a structural phase transition between the ringlike structure and other different ground state configurations occurs. The transition points are associated with a first (squares) or with a second (stars) order structural transition. In general, we observed that, at the same transition line the order of the transition (first or second order) may vary, which means that the character of the structural phase transition also depends on $\alpha$ and $\beta$.

In Fig. 14(a), the phase diagram for the cluster with $N_{f}$ $=N_{v}=5$ particles is shown. In regions I and III the different type of particles are separated in distinct rings, as can be observed in the inset of the same figure. In region II the
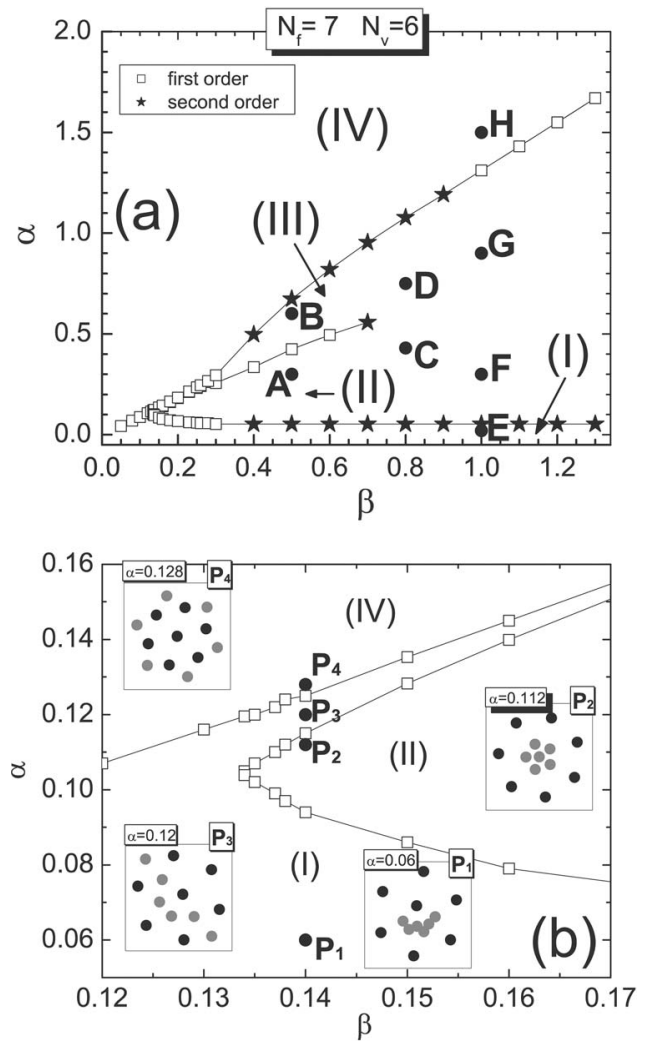

FIG. 15. (a) The same as Fig. 14, but now for the cluster with $N_{f}=7, N_{v}=6$ particles. The points $A, B, C$, and $D$ correspond to the configurations shown in Figs. 16(a), 16(b), 16(c), and 16(d), respectively; while the points $E, F, G$, and $H$ correspond to the configurations presented in Figs. 1(e), 1(f), 1(g), and 1(h), respectively. (b) Enlargement of the phase diagram (a) near $\beta \approx 0.15$. The ground state configurations shown as inset correspond to the points $P_{1}, P_{2}, P_{3}$, and $P_{4}$.

ringlike structure is not present and a mixing of the two species of particles is observed. By mixing, we mean an arrangement of particles in which both type of particles can be found, e.g., at the same distance from the center of the confinement potential. With other words, we can find both type of particles in a given shell [see Fig. 1(c)]. For the cluster with $N_{f}=N_{v}=5$ particles, the change from the ringlike configuration to another one and vice versa (transitions from region I to region II and from region II to region III) is always through a structural phase transition, and the order of the transition depends on the values of $\alpha$ and $\beta$.

For the cluster with $N_{f}=7, N_{v}=6$ particles, the ringlike structure is observed in regions II and IV of Fig. 15(a). As in the previous case, a first or a second order structural phase transition can be observed at the same transition line. Changes between regions I and II, and between regions III and IV are always characterized by a structural phase transition. However, the transition between the ringlike configuration (region II) and a different arrangement of the particles (regions III) is not always characterized by a structural phase transition. As can be observed in Fig. 15(a), the transition line characterizing the change from region II to region III is no longer present for $\beta>0.7$. Such a behavior means that the system passes from the ringlike structure to a different mini- 

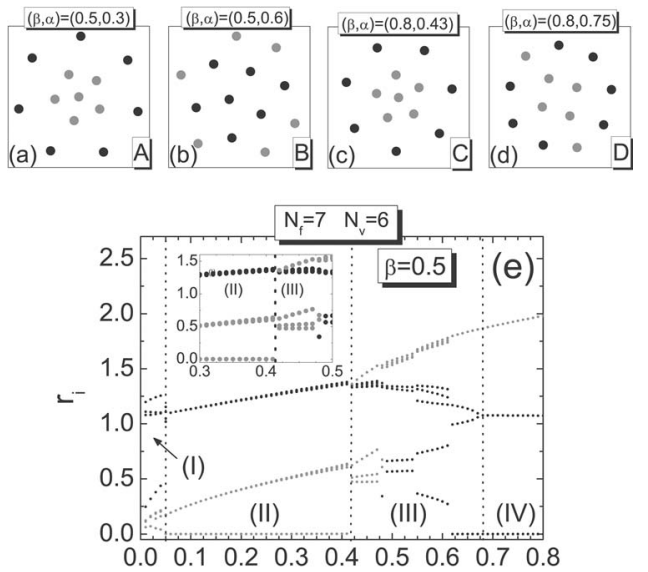

$\alpha$

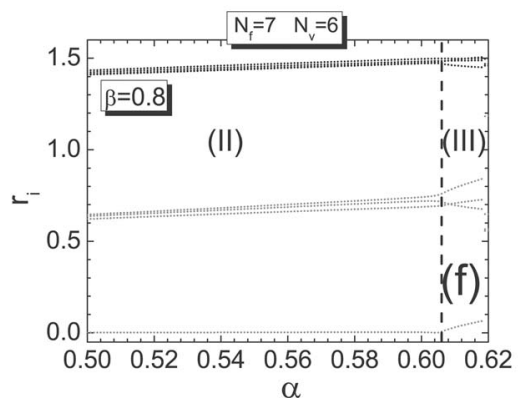

FIG. 16. Ground state configurations for the cluster with $N_{f}$ =7, $N_{v}=6$ particles in the points (a) $A$, (b) $B$, (c) $C$, and (d) $D$, shown in Fig. 15. The distances of each particle to the center of the confinement potential as a function of $\alpha$ are shown in (e) and (f) for the cases $\beta=0.5$ and 0.8 , respectively.

mum energy configuration with no discontinuity in the first or the second derivative of the energy with respect to $\alpha$ or $\beta$. The point $(\alpha, \beta)=(0.558,0.7)$ is a critical point where we have a second order transition. In Fig. 16(a)-16(d), we present examples of the configurations in the regions II and III at the points $A(0.5,0.3), B(0.5,0.6), C(0.8,0.43)$, and $D(0.8,0.75)$.

Details of how the distance of each particle to the center of the confinement potential changes as a function of $\alpha$, in regions II and III, are presented in Figs. 16(e) and 16(f), for the cases $\beta=0.5$ and 0.8 , respectively. In the case $\beta=0.5$ [Fig. 16(e)], the transition $A \rightarrow B$ is characterized by an abrupt change in the positions of the particles at $(\beta, \alpha)$ $\approx(0.5,0.42)$. Such a behavior is a typical feature of a first order structural phase transition. In the case $\beta=0.8$ [Fig. 16(f)], the transition $C \rightarrow D$ occurs without abrupt change in the positions of the particles when the system loses its ringlike configuration $(\alpha \approx 0.606)$. As in Fig. 2(b), the dashed line in Fig. 16(f) indicates the change in the ground state configuration, from the ringlike structure to a different one, with no discontinuity in the derivatives of the energy with respect to $\alpha$. More examples of the ground state configurations at the different regions of the $(\alpha, \beta)$ phase diagram, for the cluster with $N_{f}=7, N_{v}=6$ particles, are shown in Figs. 1(e), 1(f), 1(g), and 1(h) and such configurations correspond to the points $E, F, G$, and $H$ in Fig. 15(a).

The results presented in Fig. 14(a) and in Fig. 15(a) also indicate that for small values of $\alpha$ and $\beta$, the transition lines merge into one another when $\alpha \rightarrow 0$ and $\beta \rightarrow 0$. In fact, this is the case only for the cluster with $N_{f}=N_{v}=5$ particles, as shown in Fig. 14(b). The cluster with $N_{f}=7, N_{v}=6$ particles is more complex, since it presents more than two transition lines. In the limit $\alpha \rightarrow 0$ and $\beta \rightarrow 0$, the transition lines that separate regions I and II and regions II and III merge into the other at $(\beta, \alpha) \approx(0.13,0.1)$ [Fig. $15(\mathrm{~b})]$. For smaller values of $\beta$ there is only one transition line, which separates the two regions I and IV. In region IV the ground state configuration is characterized by the presence of a shell structure, while in region I a different arrangement of the particles is observed [Fig. 1(e); point $E$ of Fig. 15(a)].

It is worthwhile to comment that regions I and III are, in fact, the same. As can be seen in Fig. 15(b), there is a very narrow channel (point $P_{3}$ ) which connects both regions I and III. Thus the system presents an interesting reentrant ordered configuration with respect to the parameter $\alpha$. For example, the ground state configurations presented as insets in Fig. 15(b) correspond to the points $P_{1}, P_{2}, P_{3}$, and $P_{4}$ in the same figure. In region I the particles are arranged in a very asymmetric mixed configuration (point $P_{1}$ ). An increase in the parameter $\alpha$ leads the system to an ordered ringlike configuration (point $P_{2}$ ). A further increase in $\alpha$ destroys such a configuration, and puts the particles again in an asymmetric arrangement (point $P_{3}$ ). Finally, in region IV, the ringlike configuration is again restored (point $P_{4}$ ), and no change in the arrangement of the particles, for higher values of $\alpha$, is observed. Note also that the positions of the particles with different charges are already inverted in this region.

As shown, several configurations could be obtained as function of the parameters $\alpha$ and $\beta$. Different from the ringlike structure, we observed that for some values of $(\alpha, \beta)$ the two types of particles can be arranged in asymmetrical and mixed configurations. It is possible to quantify the different minimum energy configurations in an alternative way by defining a single new parameter, as will be shown in the next paragraphs.

As pointed out by Landau and Lifshitz [19], it is possible to describe quantitatively the change in the structure of the cluster when it passes through the structural phase transition point by an order parameter.

Since the present system is always considered at zero temperature, only a solid phase is observed. However, we can make here an analogy with the order parameter defined by Landau. Our order parameter (labeled as $\eta$ ) takes zero value when the different types of particles into the cluster are separated in distinct shells, as in a perfect ringlike configuration [Figs. 1(a) and 1(f)], and it takes a nonzero value when such an arrangement is destroyed. Thus, in the case of a mixed configuration the order parameter will be nonzero. As already commented, a mixed configuration is considered, e.g., when particles of both types are placed on the same shell [e.g., see Figs. 1(c) and 1(k), and Figs. 7(f) and 7(j)].

Before we can define our order parameter we first have to introduce another quantity. The center of mass for each of the clusters with one type of particle is defined as

$$
\mathbf{R}_{c . m .}^{j}=\frac{1}{N_{j}} \sum_{i=1}^{N_{j}} \mathbf{r}_{i}^{j},
$$

where $j=f, v$ labels particles with fixed charge and with variable charge, respectively, and $N_{j}$ represents the number 

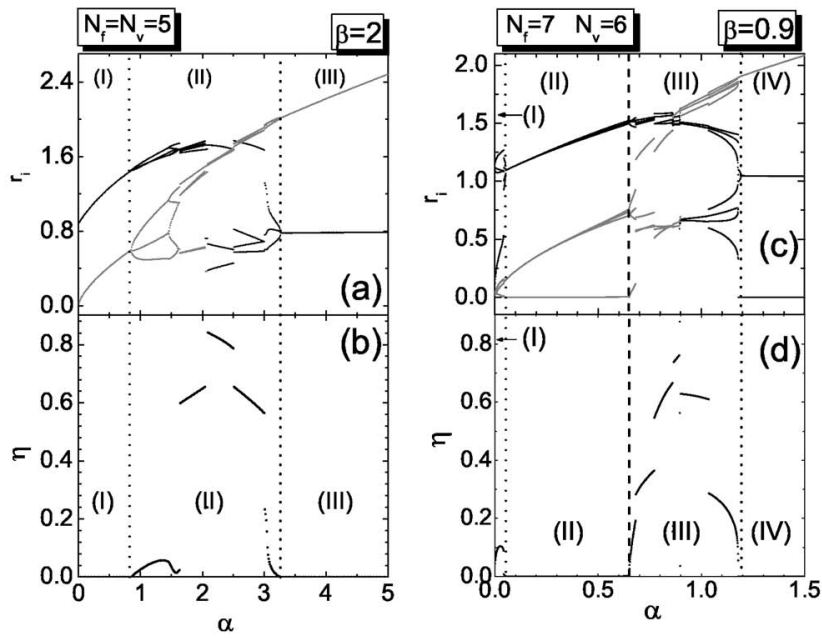

of particles with fixed or variable charge. When particles of different types are separated in distinct shells, we noticed that a symmetric configuration (with respect to the center of the confinement potential) is obtained for each type of particle [e. g., see Figs. 1(a) and 1(h)]. In this case, the center of mass for each cluster (with fixed and variable charge) is located at the center of the trap. On the other hand, when a mixing of the two type of particles is observed [e.g., see Figs. $1(\mathrm{~g})$ and $1(\mathrm{n})]$, the clusters with each type of particle present an asymmetric configuration. In this case, the center of mass for each of the two set of particles is no longer on the center. Now, we are able to define an order parameter which gives the information if the ordered state of the entire cluster corresponds to a mixed configuration or not. The order parameter is defined as the sum of the center of mass for each of the two set of particles, i.e.,

$$
\eta=R_{c . m .}^{f}+R_{c . m .}^{v} .
$$

When the entire cluster has a perfect ringlike configuration (where each ring is formed only by one type of particle) the order parameter is zero.

As an example, we show in Fig. 17 the order parameter as a function of $\alpha$ for clusters with different number of particles. More specifically, in Figs. 17(a) and 17(b), the distance of each particle to the center of the confinement potential $\left(r_{i}\right)$ and the order parameter $(\eta)$ for the cluster with $N_{f}$ $=N_{v}=5$ particles $(\beta=2.0)$ are presented. Notice that in the $\alpha$ intervals corresponding to the perfect ringlike configuration (regions I and III), the order parameter is zero. For other configurations, a nonzero value for $\eta$ is obtained (region II).

The distance of each particle to the center of the confinement potential and the order parameter for the cluster with $N_{f}=7, N_{v}=6$ particles are shown in Figs. 17(c) and 17(d) and, respectively. A perfect ringlike configuration is observed only in part of region II, and in region IV. When this is the case, the order parameter is zero. The perfect ringlike structure in region II starts to be destroyed at $\alpha \gtrsim 0.4$. Note that the distances of the particles to the center of the confinement potential start to become slightly different from each other [Fig. 17(e)]. The ground state configuration changes continuously, and no structural phase transition is observed [see Fig.

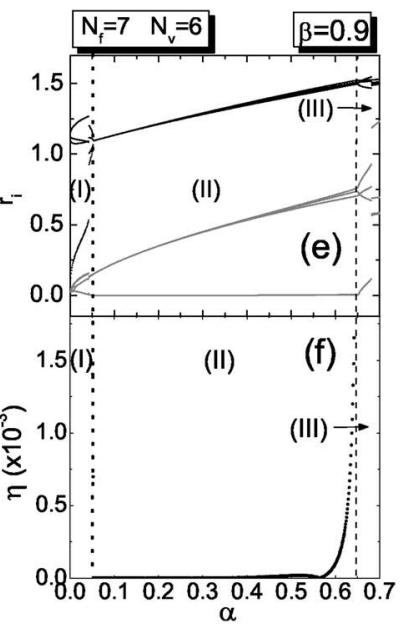

FIG. 17. (a) The distance of each particle to the center of the confinement potential and (b) the order parameter defined in Eq. (5) as a function of $\alpha$ for the cluster with $N_{f}=N_{v}=5$ particles $(\beta=2)$, and similar in (c) and (d) for $N_{f}$ $=7, N_{v}=6$ particles $(\beta=0.9)$. An enlargement of this last case is shown in (e) and (f). 15(a)]. As a consequence, for $\alpha \gtrsim 0.4$ the order parameter also starts to become nonzero [Fig. 17(f)].

The order parameter is also sensitive to structural phase transitions, especially in the case of first order transitions. In such cases, there is a discontinuity in the order parameter, as expected. For second order structural phase transitions, the order parameter exhibits no discontinuity, but an abrupt change in its behavior [e.g., see the behavior of $\eta$ around $\alpha \approx 0.8$ and $\alpha \approx 3.2$ in Fig. 17(b)].

\section{CONCLUSIONS}

In summary, we studied the structure and normal mode spectra of two-dimensional clusters containing two types of charged particles when they have different charges and/or masses. The ground state configuration as well as the normal mode spectra are strongly dependent on the charge and on the mass of both types of particles. The particles do not always arrange themselves in a ringlike configuration. However, in some range of values of the parameter $\alpha(\beta)$ there is a clear separation of the particles with different charge (mass) into distinct shells. Modifications in the structure of the system are characterized by first or second order structural phase transitions. In spite of the circular symmetry of the confinement potential, remarkable asymmetrical configurations were found.

The normal modes present intrinsic features which depend on the parameters $\alpha$ and $\beta$. It was shown that the normal modes are mainly determined by $\alpha$ and $\beta$, and not only by the number of particles in the cluster $\left(N_{f}\right.$ and $\left.N_{v}\right)$ and the ground state configuration. The center of mass and the breathing modes, observed in a system with parabolic confinement of identical particles, are observed only in the case in which the particles have different charges, but equal masses. If the masses of both types of particles are different, there is a symmetry breaking in the confinement parabolic potential, which destroys the center of mass and the breathing modes.

Commensurate ground state configurations appear here as a very stable arrangement of particles. The lowest nonzero frequency in such cases is typically three to four orders of 
magnitude larger than the lowest nonzero frequency observed in cases of noncommensurate configurations. This behavior is observed in the case of particles with different charges, as well as in the case of particles with distinct masses.

A quantitative study of the mixing between the two types of particles was also analyzed through an $(\alpha, \beta)$ phase diagram. We showed that there is a first or a second order structural phase transition when the cluster transits from a ringlike (mixed) structure to a mixed (ringlike) configuration. The order of the transition depends on the parameters $\alpha$ and $\beta$, and a reentrant ordered ringlike configuration is observed for specific values of such parameters. The mixing between the different types of particles was quantitatively characterized by a one dimensional order parameter, which is very sensitive to first or second order structural transitions.

\section{ACKNOWLEDGMENTS}

F.F.M., R.N.C.F., and G.A.F. were supported by the Brazilian National Research Councils (CAPES, CNPq). Part of this work was supported by the Flemish Science Foundation (FWO-Vl), the Belgian Science Policy, and the "Onderzoeksraad van de Universiteit Antwerpen” (GOA).
[1] A. V. Filinov, M. Bonitz, and Yu. E. Lozovik, Phys. Rev. Lett. 86, 3851 (2001).

[2] R. S. Crandall and R. Williams, Phys. Lett. 34A, 404 (1971).

[3] C. C. Grimes and G. Adams, Phys. Rev. Lett. 42, 795 (1979).

[4] M. Golosovsky, Y. Saado, and D. Davidov, Phys. Rev. E 65, 061405 (2002).

[5] B. G. Levi, Phys. Today 41, 17 (1988).

[6] J. H. Chu and L. I, Phys. Rev. Lett. 72, 4009 (1994).

[7] V. M. Bedanov and F. M. Peeters, Phys. Rev. B 49, 2667 (1994).

[8] V. A. Schweigert and F. M. Peeters, Phys. Rev. B 51, 7700 (1995).

[9] G. A. Farias and F. M. Peeters, Solid State Commun. 100, 711 (1996); W. P. Ferreira, A. Matulis, G. A. Farias, and F. M. Peeters, Phys. Rev. E 67, 046601 (2003); W. P. Ferreira, F. M. Peeters, and G. A. Farias, Phys. Rev. E 68, 066405 (2003).

[10] J. A. Drocco, C. J. Olson Reichhardt, C. Reichhardt, and B. Jankó, Phys. Rev. E 68, 060401(R) (2003).
[11] K. Nelissen, B. Partoens, and F. M. Peeters, Phys. Rev. E 69, 046605 (2004).

[12] B. A. Grzybowski, X. Jiang, H. A. Stone, and G. M. Whitesides, Phys. Rev. E 64, 011603 (2001).

[13] A. Melzer, Phys. Rev. E 67, 016411 (2003).

[14] Bin Liu, K. Avinash, and J. Goree, Phys. Rev. Lett. 91, 255003 (2003)

[15] N. Metropolis, A. W. Rosenbluth, M. N. Rosenbluth, A. M. Teller, and E. Teller, J. Chem. Phys. 21, 1087 (1953).

[16] B. Partoens and F. M. Peeters, J. Phys.: Condens. Matter 9, 5383 (1997).

[17] I. V. Schweigert, V. A. Schweigert, and F. M. Peeters, Phys. Rev. Lett. 84, 4381 (2000).

[18] B. Partoens, V. A. Schweigert, and F. M. Peeters, Phys. Rev. Lett. 79, 3990 (1997).

[19] L. D. Landau and E. M. Lifshitz, Statistical Physics (Pergamon Press, London, 1980). 